\title{
The Review of Patients' Quality of Life With Chronic Kidney Failure
}

\author{
Endang Sunariyanti ${ }^{1,{ }^{*}}$ Restu Nur Hasanah Haris ${ }^{2}$ Trisna Lestari ${ }^{3}$ Nur'aini $^{4}$ Sefi Megawati ${ }^{5}$ \\ 1, 3, 4, 5 Department of Pharmacology and Clinical Pharmacy, Faculty of Pharmacy, Sekolah Tinggi Farmasi Muhammadiyah \\ Tangerang, Tangerang, Indonesia \\ ${ }^{2}$ Department of Pharmacology and Clinical Pharmacy, Faculty of Pharmacy, ITK Avicena Kendari, Kendari, Indonesia \\ *Corresponding author.Email: endangsunariyanti@gmail.com
}

\begin{abstract}
Chronic kidney failure is a disease with a global prevalece of height. The high incedence of chronic kidney failure requires special care to improve the quality of life is the state where a person feels compertabel and saitisfied in everyday life, including physical, emotional, social and spiritual functions. Limitation of all activities, both physical and psychological, is often the cause of the decreased quality of life of patients with chronic kidney failure. This article is a systematic review that discusses the quality of life of patients with chronic kidney failure. The search for articles was carried out during2 months (April-June 2020) by the author's team through google scholar and pubmed using several appropriate keywords such as: Health Related Quality of Life (HRQoL), Chronic Kidney Disease (CKD), Hemodialysis and Kidney Disease Quality of Life.Original research articles that discuss QoL of CKD patients and published in the 2010-2020 period are inclusion criteria. PRISMA diagrams (Preferred reporting items for systematic reviews) are used to assist in the selection of articles that fit the established inclusion and exclusion criteria. The results of the systematic review showed that as many as 87 articles were screened according to the study of the abstract and title. The team of authors obtained 38 articles according to the inclusion criteria, while the other 49 articles were exclusion criteria. This systematic review shows that the quality of life in patients with chronic kidney failure is decreasing in various domains. Patients experience decreased quality of life in term of finances, work, physical limitions, mental and social roles.This situations is exacerbated if the patient is undergoing hemodialysis therapy. Physical limitations is the worst domain and has the lowest score compared to other domains. Older age, comorbid diabetes mellitus, higher stage of CKD and longer durataion of haemodyalisations according to influnce the quality of life of chronic kidney failure.
\end{abstract}

\section{Keywords: Health Related Quality of Life (HRQoL), Chronic Kidney Disease (CKD), Hemodialysis dan Kidney Disease Quality of Life}

\section{INTRODUCTION}

Chronic Kidney Failure Disease is a disease with a high prevalence rate and a global health problem. The United State Renal Disease Data System (USRDS) in the United States reports that the increase in the incidence of chronic kidney disease in the United States is increasing by $20-25 \%$ each year. Global Burden of Disease (2010) data on chronic kidney failure ranks 27 in the world as the largest cause of death. Hemodyalysis is one of the treatments for kidney failure with the hope of improving the quality of life (QoL) of the patient. Quality of life is a condotions where a person can feel satisfactions in all daily activites in carrying out physical activities, control mentally and feel satisfied in everyday social roles $(\mathrm{WHO}, 2016)^{39}$.

A person can be said to have a good quality of life when he is physically able, mentally healthy and has a role in his social society.Previous study have show that a large proportion of patient with chronic renal failure show deteriorattions in quality of life. Many of them feel that the quality of their lives is worsening because of limited daily physical activities and poor mental / emotoonal states when yhe diagnosis of illness is is receivad (wild, 2019) (0. The $^{4}$ quality of life (QoL) of chronic kidney failure patients decreases due to emotional instability and has begun to surrender to the disease, which also affects the physical domain and social interactions in everyday life. Chen $(2016)^{41}$ in a systematic review wrote that several factors that contribute to the deterioration of the quality of life of patients with chronic kidney failure are comorbid factors and complications suffered, age, hemodyalysis treatment, gender and disease severity. Kidney failure greatly affects the physical and mental status where the patient feels physically limited compared to emotional factors. The artical provides informations to readers regarding the quality of life of patients with cronic dissorders and the factrors that influnce it.

\section{RESEARCH METHOD}

The article discusses the quality of life (QoL) of patients with chronic kidney failure. The reseacher tries to describe chronic kidney failure that affects the quality of life and sees the limitations of patients in everyday life. The PRISMA diagram (Preferred reporting item for systematic reviews) is used by the reseacher to facilitate the search and selection of 
articles to be shown (Figure I). The articles reviewed had met the inclusion and exclusion criteria stated in the data extract.

\section{SEARCH STRATEGY}

Search for articles was carried out for approximately 2 months, from April 10 to June 2020. To facilitate the search, the appropriate keywords were used, namely: Health Related Quality of Life (HRQoL), Chronic Kidney Disease (CKD), Hemodialysis and Kidney Disease Quality of Life. Article searches were carried out on Google scholar (100 articles) and Pubmed (22 articles). The reseacher identifies articles by screening titles and abstracts and then adjusts them to the inclusion and exclusion criteria that have been set.

\section{INCLUSION AND EXCLUSION CRITERIA}

The existing inclusion and exclusion criteria make it easier for the author to get the appropriate and expected articles. An original research type article containing QoL of CKD patients, published 2010-2020 (English \& Indonesian) is an inclusion criteria. Inclusion and exclusion criteria can be seen in table 1.
Table 1. Inclusion and Exclusion Criteria

\begin{tabular}{|l|l|}
\hline Inclusion Criteria & Exclusion Criteria \\
\hline $\begin{array}{l}\text { 1. Original research } \\
\text { 2. Articles in English- } \\
\text { Indonesian }\end{array}$ & $\begin{array}{l}\text { 1. Systematic review, } \\
\text { editorial atau report } \\
\text { articles }\end{array}$ \\
$\begin{array}{l}\text { 3. Published in late 10 years } \\
\text { (2010-2020) }\end{array}$ & $\begin{array}{l}\text { 2. Nofull text } \\
\text { 3. Using generic or specific not discuss QoL } \\
\text { QoL instrument }\end{array}$ \\
\hline
\end{tabular}

\section{EXTRACT DATA}

At the screening stage, the reseachers identified the articles according to the title and abstract, which obtained 87 articles. The results of our review that 38 articles met the inclusion criteria while the other 49 articles were exclusion criteria. Articles are included in a table containing the characteristics of data identification: Table (I) research study, research method design, data collection location, number of samples, QoL instrument used and instrument validation. Table (II): score / utility, impact of CKD on patient QoL and description of Comorbid and complications suffered.
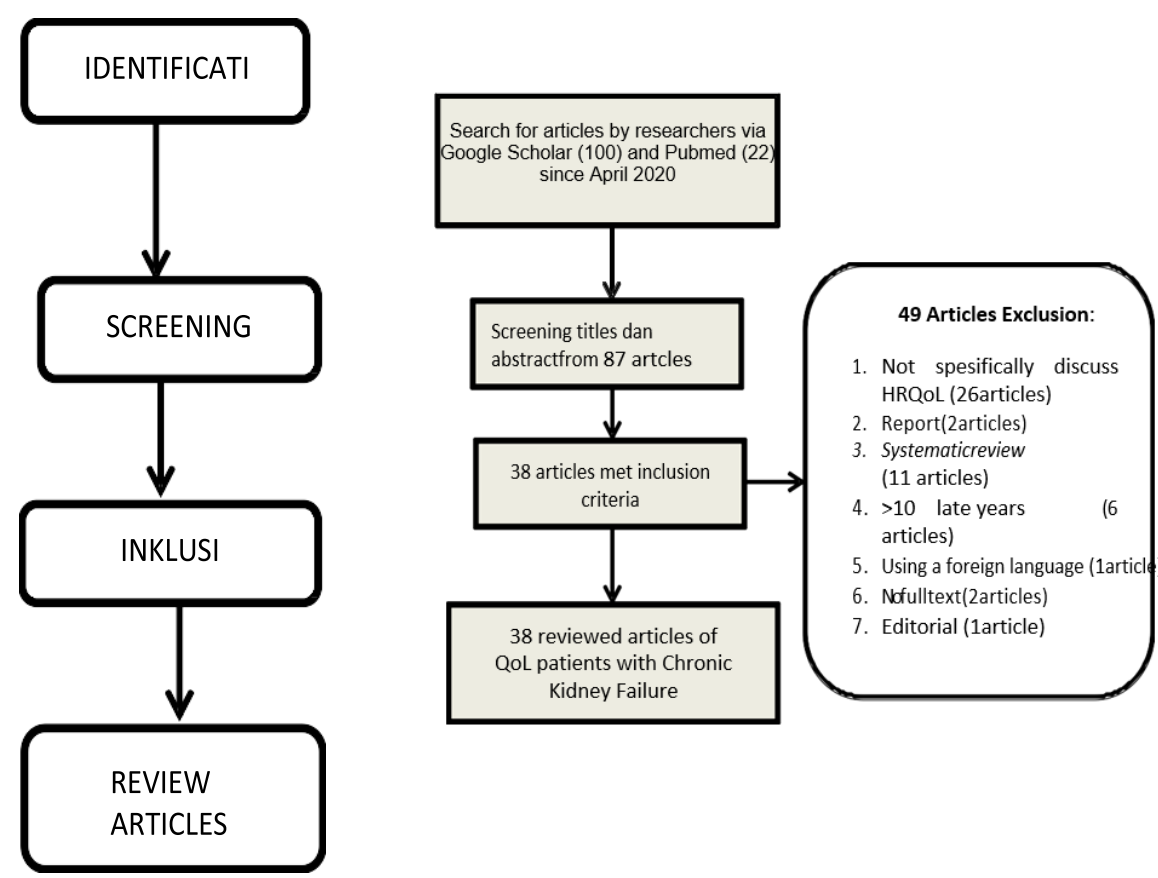

Figure I. Prisma Diagram 
In this systematic review, the PRISMA diagram shows that 49 articles were excluded. A total of 26 articles did not discuss QoL in CKD patients specifically, 11 articles were systematic review, editorial (6 articles), report ( 2 articles). 6 articles published $>10$ years, 2 articles are not full text and in foreign languages (Figure I).

In our review, $65.7 \%$ of the articles used a cross-sectional study design, while the other articles used an observational and descriptive study design. The sampling locations in this systematic review came from 19 countries (India, Sweden, Nigeria, Athens, Saudi Arabia, Bulgaria, Indonesia, Belgium, Denmark, Iran, China, Malaysia, Spain, US, Singapore, Palestine, Portugal, Australia and Korea. Our review assessed that $57.8 \%$ of the sampling was carried out in hospitals and the rest were in the hemodialysis clinic. The instruments used to measure the quality of life also varied using generic and specific instruments. The generic instruments used were Short From-36 (SF-36), SF- 6D (Short From-6 Dimension), WHOQOL-BREF (WHO-Quality of Life-BREF) and EQ5D-5L (European Quality of Life-Five Dimension-5L) and PedsQoL.The SF-36 instrument is the most generic instrument. is widely used to assess the quality of life of patients with Chronic Kidney Failure (15.7\%). However, the reseachers assess, the use of specific instruments is more widely used in this systematic review. uality of Life). Several other articles assessed the quality of life of CKD patients using PHC and MHC (physical and mental health) instruments, BMVQOLI-15, KINDL and BDI. In measuring QoL using instruments, psychometric properties testing is important to ensure its validity and reliability of the instruments used. Our review shows that only a portion of the articles tested psychometric properties (18.4\%). The validity test is the construct validity-convergent validity, while the reliability is carried out using the internal consistency with the Cronbach alpha value (>0.7). 


\begin{tabular}{|c|c|c|c|c|c|c|c|}
\hline No & $\begin{array}{l}\text { Studies/ } \\
\text { Year }\end{array}$ & Country & Methods & Settings & Sample size & Instrument & Validation \\
\hline 1 & Cruz (2011) & $\begin{array}{c}\text { San Paulo } \\
\text { Brazil }\end{array}$ & $\begin{array}{l}\text { Cross- } \\
\text { sectional }\end{array}$ & $\begin{array}{l}\text { Out patient at a neprology } \\
\text { clinic, Brazil }\end{array}$ & 155 & SF-36 & NA \\
\hline 2 & Turlapati $(2015)^{2}$ & India & $\begin{array}{l}\text { observational } \\
\text { prospective }\end{array}$ & $\begin{array}{llll}\begin{array}{l}\text { Patients in } \\
\text { hospital }\end{array} & \text { tertiary } & \text { care } \\
\end{array}$ & 99 & SF-36 & NA \\
\hline 3 & $\begin{array}{l}\text { Vankateswaramrt } \\
\text { hy }(2018)^{3}\end{array}$ & India & $\begin{array}{l}\text { Observational } \\
\text { \& prospective } \\
\text { Study }\end{array}$ & $\begin{array}{l}\text { Patients in nephrology } \\
\text { department of a tertiary care } \\
\text { hospital }\end{array}$ & 200 & KDQOL-SF & NA \\
\hline 4 & Berger $(2016)^{4}$ & Brazil & $\begin{array}{l}\text { Descriptive } \\
\text { prospective } \\
\text { study }\end{array}$ & $\begin{array}{l}\text { Kidney failure patient in } 4 \\
\text { Clinics in, Brazil }\end{array}$ & 286 & KDQOL-SF-36 & NA \\
\hline 5 & Pagels $(2012)^{5}$ & Sweden & $\begin{array}{c}\text { Cross- } \\
\text { sectional }\end{array}$ & $\begin{array}{l}\text { Outpatients eight nephrology } \\
\text { units }\end{array}$ & 535 & SF-36 & NA \\
\hline 6 & Jesus $(2018)^{6}$ & Brazil & $\begin{array}{c}\text { Cross- } \\
\text { sectional }\end{array}$ & $\begin{array}{l}\text { Patients in public clinic and a } \\
\text { private hemodialysis clinic. }\end{array}$ & 114 & $\begin{array}{l}\text { WHOQOL- } \\
\text { BREF }\end{array}$ & NA \\
\hline 7 & Murali $(2014)^{7}$ & India & $\begin{array}{l}\text { observational } \\
\text { prospective, }\end{array}$ & $\begin{array}{l}\text { Patients two tertiary care } \\
\text { clinics in ambulatory }\end{array}$ & 50 & KDQOL-SF & NA \\
\hline 8 & Ayanda $(2014)^{8}$ & Nigeria & $\begin{array}{c}\text { Cross- } \\
\text { sectional }\end{array}$ & $\begin{array}{l}\text { Patients in University of llorin } \\
\text { Teaching Hospital (UITH), }\end{array}$ & 113 & $\begin{array}{l}\text { WHOQOL- } \\
\text { BREF }\end{array}$ & $\overline{N A}$ \\
\hline 9 & Georgia $(2017)^{9}$ & Athens & $\begin{array}{c}\text { descriptive } \\
\text { study }\end{array}$ & $\begin{array}{l}\text { Patients in Dialysis Unit in a } \\
\text { public hospital }\end{array}$ & 70 & $\begin{array}{l}\text { WHOQOL- } \\
\text { BREF }\end{array}$ & NA \\
\hline 10 & Wakeel $(2012)^{10}$ & Saudi Arabia & $\begin{array}{l}\text { Cross- } \\
\text { sectional }\end{array}$ & $\begin{array}{l}\text { Patient Undergoing } \\
\text { hemodialiasis at Rs Khalid } \\
\text { Saudi Arabia. }\end{array}$ & 200 & KDQOL-SF & NA \\
\hline 11 & $\begin{array}{l}\text { Abdhelghany } \\
{ }^{11}(2016)\end{array}$ & $\begin{array}{l}\text { Alexandria, } \\
\text { Egypt }\end{array}$ & $\begin{array}{c}\text { Cross- } \\
\text { sectional; } \\
\text { Descriptive }\end{array}$ & $\begin{array}{l}\text { Patients hemodialysis unit of } \\
\text { Karmouz Health Insurance } \\
\text { Hospital }\end{array}$ & 81 & KDQOL-SF-36 & Construct Validity \\
\hline 12 & Dimova $(2016)^{12}$ & Bulagaria & $\begin{array}{l}\text { Multi-center } \\
\text { cross- } \\
\text { sectional. }\end{array}$ & $\begin{array}{l}\text { Patients hemodialysis centers } \\
\text { across the country }\end{array}$ & 263 & B-MVQOLI-15 & $\begin{array}{l}\text { Internal } \\
\text { consistency }(0.73) \\
\& \text { convergent } \\
\text { validity }(0.385)\end{array}$ \\
\hline
\end{tabular}




\begin{tabular}{|c|c|c|c|c|c|c|c|}
\hline No & $\begin{array}{c}\text { Studies/ } \\
\text { Year }\end{array}$ & Country & Methods & $\begin{array}{l}\text { Setti } \\
\text { ngs }\end{array}$ & Sample size & Instrument & Validation \\
\hline 13 & Saputri $(2018)^{13}$ & Indonesia & $\begin{array}{l}\text { cross- } \\
\text { sectional. }\end{array}$ & $\begin{array}{l}\text { Hemodialysis Patients at } \\
\text { the pringsewu District } \\
\text { general Hospital.Indonesia }\end{array}$ & 117 & KDQOL-SFTM & $\begin{array}{c}\text { Internal consistency } \\
\& \text { coefficient } \\
\text { reliability } \\
\text { (Cronbach's alpha) } \\
(0.941)(0.937)\end{array}$ \\
\hline 14 & Costa $(2016)^{14}$ & Brazil & $\begin{array}{c}\text { descriptive \& } \\
\text { transversal } \\
\text { study, }\end{array}$ & $\begin{array}{l}\text { Patients in the Dialysis } \\
\text { Center } \\
\text { of Cajazeiras (CHC), }\end{array}$ & 50 & $\begin{array}{l}\text { WHOQOL- } \\
\text { BREF }\end{array}$ & NA \\
\hline 15 & Poppe $(2013)^{15}$ & Belgium & $\begin{array}{l}\text { Cross- } \\
\text { Sectional }\end{array}$ & $\begin{array}{l}\text { Outpatient clinic of } \\
\text { the } \\
\text { Nephrology department } \\
\text { ofthe } \\
\text { Ghent University Hospital, }\end{array}$ & 155 & $\begin{array}{l}\text { SF-36 } \\
\text { PHQL and } \\
\text { MHQL) }\end{array}$ & $\begin{array}{l}\text { Cronbach's alpha } \\
\text { coefficients }(0.88)\end{array}$ \\
\hline 16 & Knudsen $(2016)^{16}$ & Denmark. & $\begin{array}{l}\text { Cross- } \\
\text { Sectional }\end{array}$ & $\begin{array}{l}\text { Renal Failure Pateint In } \\
\text { Cardiology departement, } \\
\text { Nephrologist who is at Rs. } \\
\text { Nordsjaellands. }\end{array}$ & 152 & $\overline{\mathrm{KDQOL}}$ & Reliability \\
\hline 17 & $\begin{array}{c}\text { Veerappan } \\
(2020)^{17} \\
\end{array}$ & India & $\begin{array}{c}\text { Cross- } \\
\text { Sectional } \\
\end{array}$ & $\begin{array}{ll}\text { Patients } & \begin{array}{l}\text { in tertiary } \\
\text { care hospital }\end{array} \\
\end{array}$ & 131 & KDQOL-36 & $\overline{N A}$ \\
\hline 18 & Naderifar $(2019)^{18}$ & Iran & $\begin{array}{l}\text { Descriptive- } \\
\text { analitic }\end{array}$ & $\begin{array}{l}\text { Shahid Beheshti University } \\
\text { of Medical Sciences, }\end{array}$ & 200 & KDQOL-SF & $\begin{array}{c}\text { validity and } \\
\text { reliability } \\
\text { coefficients }(0.77) \\
(0.83) \\
\end{array}$ \\
\hline 19 & Ossama $(2020)^{19}$ & Egypt & $\begin{array}{l}\text { Cross- } \\
\text { Sectional }\end{array}$ & $\begin{array}{l}\text { dialysis unit of both Benha } \\
\text { University Hospital and } \\
\text { Benha Teaching Hospital. }\end{array}$ & 228 & KDQOL-SF36 & NA \\
\hline 20 & Rostami $(2013)^{20}$ & Iran & $\begin{array}{l}\text { Cross- } \\
\text { Sectional }\end{array}$ & $\begin{array}{ll}\text { patients } & \text { from } 132 \\
& \text { dialysis centers }\end{array}$ & 6930 & KDCS-SF & $\begin{array}{c}\text { internalconsistency } \\
\text { reliability : } \\
\text { Cronbach alphas } \\
0.43 \text { to } 0.91\end{array}$ \\
\hline 21 & $\operatorname{Pan}(2018)^{21}$ & China & $\begin{array}{l}\text { Cross- } \\
\text { Sectional }\end{array}$ & $\begin{array}{l}\text { Patients from a tertiary } \\
\text { hospital China. }\end{array}$ & 315 & $\begin{array}{l}\text { KDQOL (PCS, } \\
\text { MCS, SF-6D, } \\
\text { KDCS) }\end{array}$ & NA \\
\hline 22 & Nayana $(2016)^{22}$ & Kerala & $\begin{array}{c}\text { Cross- } \\
\text { Sectional } \\
\text { descriptive }\end{array}$ & $\begin{array}{l}\text { Patients dialysis unit of a } \\
\text { tertiary level referral } \\
\text { hospital }\end{array}$ & 50 & KDQOL & NA \\
\hline
\end{tabular}


Advances in Health Sciences Research, volume 33

\begin{tabular}{|c|c|c|c|c|c|c|c|}
\hline No & $\begin{array}{l}\text { Studies/ } \\
\text { Year }\end{array}$ & Country & Methods & Settings & Sample size & Instrument & Validation \\
\hline 23 & Bender $(2018)^{23}$ & Warsaw & $\begin{array}{c}\text { Cross- } \\
\text { Sectional }\end{array}$ & $\begin{array}{l}\text { The Clinic of Nephrology and } \\
\text { Dialysis }\end{array}$ & 100 & KDQOL-SF12 & NA \\
\hline 24 & $\begin{array}{l}\text { Sihombing } \\
(2017)^{24}\end{array}$ & Indonesia & $\begin{array}{l}\text { Multicenter } \\
\text { prospective } \\
\text { study }\end{array}$ & $\begin{array}{l}\text { Patinets from } 3 \text { big hospitals in } \\
\text { Yogyakarta }\end{array}$ & 113 & $\mathrm{KDQOL} \mathrm{SF}^{\mathrm{TM}}$ & NA \\
\hline 25 & Morales $(2018)^{25}$ & Peru & $\begin{array}{c}\text { descriptive, } \\
\text { cross- } \\
\text { sectional, } \\
\text { prospective, } \\
\text { observational } \\
\text { study }\end{array}$ & $\begin{array}{llll}\text { Kidney } & \text { Failure } & \text { Patient At } \\
\text { Cayetano } & \text { Heredia } & \text { Hospital } \\
\text { Unit, Paru } & & \end{array}$ & 79 & KINDL & NA \\
\hline 26 & Surendra $(2019)^{26}$ & Malaysia & $\begin{array}{l}\text { cross-sectional } \\
\text { study }\end{array}$ & $\begin{array}{l}\text { From the National Renal } \\
\text { Registry (NRR) }\end{array}$ & 141 & EQ-5D-3L & NA \\
\hline 27 & Montilla $(2016)^{27}$ & Spanish & & $\begin{array}{l}\text { From Health district } \\
\text { (Haemodialysis Unit at Hospital }\end{array}$ & 52 & $\begin{array}{l}\text { SF-36 } \\
\text { PHC } \\
\text { MHC }\end{array}$ & $\begin{array}{l}\text { internal consistency } \\
\text { (Cronbach's _) } \\
(0.7)(0.94) . \text { PHC, } \\
\text { Cronbach's }(0.94) \text {, } \\
\text { MHC, }(0.89)\end{array}$ \\
\hline 28 & Heidari $(2020)^{28}$ & Northern Iran & $\begin{array}{c}\text { Cross- } \\
\text { sectional }\end{array}$ & $\begin{array}{l}\text { Patients from Shahid Beheshti } \\
\text { Hospital }\end{array}$ & 154 & SF-36 & NA \\
\hline 29 & Pardede $(2019)^{29}$ & Indonesia & $\begin{array}{l}\text { Cross- } \\
\text { sectional } \\
\text { analytical } \\
\text { study }\end{array}$ & $\begin{array}{l}\text { Outpatient Care Unit of } \\
\text { Nephrology } \\
\text { Hemodialysis Unit and Inpatient } \\
\text { Care Unit of Child Health } \\
\text { Department, RSCM }\end{array}$ & 112 & PedsQL & NA \\
\hline 30 & Stein $(2012)^{30}$ & US & $\begin{array}{c}\text { Cross- } \\
\text { sectional }\end{array}$ & $\begin{array}{l}\text { Patients from dialysis unit and } \\
\text { home dialysis }\end{array}$ & 114 & KDQOL-36 & NA \\
\hline 31 & Wang $(2012)^{31}$ & Singapore & $\begin{array}{l}\text { census-style } \\
\text { survey }\end{array}$ & $\begin{array}{l}\text { from } 22 \text { dialysis centers across } \\
\text { Singapore }\end{array}$ & 1249 & KDQOL-SF & NA \\
\hline 32 & $\begin{array}{l}\text { Fructuoso } \\
(2011)^{32}\end{array}$ & Portugal & $\begin{array}{c}\text { Cross- } \\
\text { sectional }\end{array}$ & $\begin{array}{l}\text { From Nephrology Department } \\
\text { and dialysis unit }\end{array}$ & 821 & $\begin{array}{c}\text { SF-36 } \\
\text { KDQOL-SF }\end{array}$ & NA \\
\hline 33 & Zyoud $(2016)^{33}$ & Palestina & $\begin{array}{l}\text { Cross- } \\
\text { sectional }\end{array}$ & Patients From dialysis center & 267 & EQ-5D-5L & NA \\
\hline 34 & Zimbudzi $(2016)^{34}$ & Australia & single-centre, & From public teaching hospital & 81 & EQ-5D-5L & \\
\hline
\end{tabular}




\begin{tabular}{|c|c|c|c|c|c|c|c|}
\hline & & & $\begin{array}{l}\text { prospective } \\
\text { cohort study }\end{array}$ & & & & \\
\hline 35 & Negi $(2019)^{35}$ & India & $\begin{array}{c}\text { Descriptive } \\
\text { cross-sectional }\end{array}$ & $\begin{array}{l}\text { Patients dialysis unit of Institute } \\
\text { of Liver and Biliary Sciences }\end{array}$ & 70 & KDQOL-SF36 & Reliability (0.90) \\
\hline 36 & Lee $(2016)^{36}$ & Korea & $\begin{array}{c}\text { comparative } \\
\text { descriptive } \\
\text { study }\end{array}$ & $\begin{array}{l}\text { Patients from a hospital in } \\
\text { Korea dialysis unit }\end{array}$ & 150 & EQ-5D-5L & NA \\
\hline 37 & Alqahtani $(2019)^{37}$ & Saudia Arabia & cross-sectional & $\begin{array}{l}\text { From the nephrology center, } \\
\text { King Fahd Military hospital }\end{array}$ & 164 & KDQOL-SF & NA \\
\hline 38 & Teles $(2018)^{38}$ & Spain & cross-sectional & $\begin{array}{l}\text { patients from a single in- } \\
\text { hospital dialysis centre }\end{array}$ & 200 & $\begin{array}{c}\text { SF-36 } \\
\text { BDI }\end{array}$ & NA \\
\hline
\end{tabular}

SF-36 ; Short Form-36; PedsQL; Peddiatric quality of life; BDI; Beck Depression Inventory; SF-12; Short Form-12; EQ-5D-5L ; European Quality-five dimension;KDQOL; Kidney Disease Quality of Life; PHQL; Physical Health Quality of Life; MHQL;Mental Health Quality of Life; CKD; Chronic Kidney Disease; WHOQOL-BREF; WHO Quality of Life-BREF. 


\section{RESULTS AND DISCUSSIONS}

The prevalence of chronic kidney failure globally is increasing every year. United State Renal Data System (USRDDS) (2013) reported that the incidence of chronic kidney failure in the world has increased by $10-13 \%$. Chronic kidney failure is one of the diseases that ranks at the top of the high mortality rate globally. The treatment provided is a step to improve the quality of life (QoL) of patients by undergoing hemodialysis (HD) or peritonial dialysis (PD). This treatment does not necessarily cure the patient because most of the kidney failure patients experience a deteriorating quality of life when diagnosed with CKD and decreased function of other organs.Most patients with a history of kidney failure will experience worsening in various domains of life, quality of life, comfort, and self - satisfaction will be reduced in the domains of finance, work, physical limitations, emotional and social environmen. The article is a type of systematic review tries to provide information related to quality of life (QoL). Patients with kidney failure treatment will experience a decrease in the quality of life in financial, work, physical, emotional and social environment. This systematic review assesses the quality of life $(\mathrm{QoL})$ of patients with chronic renal failure measured using instruments, be it generic instruments or those that are specific to measure the quality of life of chronic kidney patients. The results of our systematic review assessed that the quality of life of patients with chronic renal failure was poor in various domains, and the quality of life decreased when undergoing hemodialysis / PD treatment. Research by Cruzz (2011) 1 states that CKD patients experience a decrease in quality of life, especially in the physical dimensions and roles in daily life, as well as in the Turlapati (2015) 2 study which was measured using a specific KDQoL (Kidney Disease Quality of Life) instrument, most patients decreased function in the physical domain. This is due to deterioration of organ functions in the patient so that all the physical activities that are usually carried out on a daily basis experience limitations since suffering from chronic kidney failure. In this systematic review we also found that, although patients were limited in their physical roles, they showed better scores in the emotional and psychological domains. Murali (2014) revealed that the mental health of patients with kidney failure is better than physical health, although it shows a deterioration in quality of life. CKD patients with a good mental state can manage their emotional feelings, make feelings more peaceful, calm and can control feelings of sadness and depression (Ossama (2020) 19. These results show that emotional and mental conditions also affect the quality of life of patients with Chronic Kidney Failure.

The prevalence of chronic gynecological failure actully affects patient with advanced age (> 60 years). Age factors actually affect the quality of life in chronic kidney failure. In this systematic review, the writer found that the human factor gave a low score compared to other sociodemographic Variables.

Ayanda's research (2014) 8 shows that age affects the score of CKD patients as well as status and level of support in social life. The patient's quality of life will decline with age. However, different results were shown. It is said that elderly patients have a higher score than young people because young people are more worried and problematic about their CKD diagnosis which negatively affects the quality of life. This is associated with better mental and emotional health among older patients than younger patients. The social life of CKD patients is limited due to limited physical activity and social roles in the community, which must routinely carry out hemodyalysis treatments and even stay for a long time in the hospital. 
Table 3.Effect of CKD on patient QoL

\begin{tabular}{|c|c|c|c|c|c|}
\hline No & Study & Instrumen & $\begin{array}{l}\text { Score } \\
\text { /Utility }\end{array}$ & HRQoL & \begin{tabular}{|l|} 
Comorbid/ \\
Complication
\end{tabular} \\
\hline 1. & $\begin{array}{l}\text { Cruz } \\
(2011)\end{array}$ & SF-36 & $\begin{array}{l}\mathrm{PF}=61.5 \\
\mathrm{PRF}=48.6 \\
\mathrm{P} \quad=59.8 \\
\mathrm{GH}=51.7 \\
\mathrm{~V} \quad=54.3 \\
\mathrm{SR}=63.7 \\
\mathrm{ER}=65.7 \\
\mathrm{MH}=64.0\end{array}$ & $\begin{array}{l}\text { CKD stage and age } \\
\text { affect the patient's QoL. QoL } \\
\text { descending on a stage / stage } \\
\text { CKD. }\end{array}$ & NA \\
\hline 2. & $\begin{array}{l}\text { Turlapati } \\
(2015\end{array}$ & SF-36 & $\begin{array}{l}\mathrm{GH}=54.55 \\
\mathrm{P}=24.14 \\
\mathrm{ER}=66.75 \\
\mathrm{~S}=45.35\end{array}$ & $\begin{array}{l}\text { Comorbids and complications } \\
\text { affect QoL . }\end{array}$ & HT,DM,CG, CP \\
\hline 3. & $\begin{array}{l}\text { Vankates } \\
\text { waramrth } \\
\text { y (2018) }\end{array}$ & KDQOL-SF & $\begin{array}{l}\mathrm{S} / \mathrm{P}=77.35 \\
\mathrm{EKD}=74.66 \\
\mathrm{BKD}=34.15 \\
\mathrm{WS}=40.1 \\
\mathrm{C} \quad=62.52 \\
\mathrm{QoLs}=75.91 \\
\mathrm{SP}=65.22 \\
\mathrm{SS}=72.81\end{array}$ & \begin{tabular}{|l|} 
Comorbids and complications \\
affects QoL None \\
differences in QoL between patients \\
who do not live HD \\
with patients undergoing \\
HD.
\end{tabular} & HT, IH, DM, AN \\
\hline 4. & $\begin{array}{l}\text { Berger } \\
(2016)\end{array}$ & KDQOL- SF36 & 60.53 & $\begin{array}{l}\text { CKD affects QoL, in which case the } \\
\text { length of stay affects the patient's } \\
\text { QoL }\end{array}$ & $\mathrm{DM}$ \\
\hline 5. & $\begin{array}{l}\text { Pagels } \\
(2012)\end{array}$ & SF-36 & $\begin{array}{l}\mathrm{PF}=49.2 \\
\mathrm{PRF}=22.1 \\
\mathrm{P} \quad=55.7 \\
\mathrm{GH}=40.2 \\
\mathrm{~V} \quad=36.5 \\
\mathrm{SR}=57 \\
\mathrm{ER}=34.6 \\
\mathrm{MH}=62.9\end{array}$ & $\begin{array}{l}\text { QoL decreased on } \\
\text { stages / stage CKD, there are } \\
\text { comorbid affects } \\
\text { QoL patients }\end{array}$ & HT, DM \\
\hline 6. & $\begin{array}{l}\text { Jesus } \\
(2018)\end{array}$ & $\begin{array}{l}\text { WHOQOL- } \\
\text { BREF }\end{array}$ & $\begin{array}{l}\mathrm{RQ} 1=3.88 \\
\mathrm{RQ} 2=3.47 \\
\mathrm{PD}=56.64 \\
\mathrm{PsD}=67.5 \\
\mathrm{SD}=70.67\end{array}$ & $\begin{array}{l}\text { CKD affects QoL. } \\
\text { Patients are limited to domains } \\
\text { physical and psychological. }\end{array}$ & NA \\
\hline
\end{tabular}




\begin{tabular}{|c|c|c|c|c|c|}
\hline & & & $\mathrm{ED}=62.44$ & & \\
\hline 7. & $\begin{array}{l}\text { Murali } \\
(2014) 7\end{array}$ & KDQOL-SF & 71.93 & $\begin{array}{l}\text { Patients with CKD have poor QoL, } \\
\text { the physical health domain being the } \\
\text { most influential. Comorbids affect the } \\
\text { patient's QoL }\end{array}$ & $\begin{array}{l}\text { DM, CG, RT,PU, } \\
\text { CPD }\end{array}$ \\
\hline 8. & $\begin{array}{l}\text { Ayanda } \\
(2014) 8\end{array}$ & $\begin{array}{l}\text { WHOQOL- } \\
\text { BREF }\end{array}$ & NA & $\begin{array}{l}\text { Comorbid and CKD stage } \\
\text { affect the patient's QoL. }\end{array}$ & $\begin{array}{l}\text { Kardiovaskuler, } \\
\text { DM }\end{array}$ \\
\hline 9. & $\begin{array}{l}\text { Georgia } \\
(2017) 9\end{array}$ & $\begin{array}{l}\text { WHOQOL- } \\
\text { BREF }\end{array}$ & $\begin{array}{ll}\mathrm{PD}= & 12.90 \\
\mathrm{PsD}=13.98 & \mathrm{SD} \\
=14.68 & \\
\mathrm{ED}=14.15 & \end{array}$ & Age, marital status affect QoL & NA \\
\hline 10. & $\begin{array}{c}\text { Wakeel } \\
(2012) 10\end{array}$ & KDQOL-SF & $\begin{array}{l}\mathrm{HD}=49.5 \\
\mathrm{PD}=61.3\end{array}$ & $\begin{array}{l}\text { Patients with HD have a low QoL } \\
\text { score, and the age and duration of HD } \\
\text { influence. }\end{array}$ & NA \\
\hline 11. & $\begin{array}{l}\text { Abdhelgh } \\
\text { any } \\
26(2016)\end{array}$ & $\begin{array}{l}\text { KDQOL- } \\
\text { SF36 }\end{array}$ & $\begin{array}{l}\mathrm{S} / \mathrm{P}=49.97 \\
\mathrm{EKD}=51.04 \\
\mathrm{BKD}=25.62 \\
\mathrm{WS}=30.86 \\
\mathrm{C} \quad=62.96\end{array}$ & $\begin{array}{l}\text { Patients with HD have } \\
\text { low QoL score. Comorbid } \\
\text { affect the patient's QoL }\end{array}$ & AN, DM,HP \\
\hline & & & $\begin{array}{l}\text { QoLs }=71.52 \\
\text { SP }=50.22 \\
\text { SS }=72.22 \\
\text { SF-36=47.22 }\end{array}$ & & \\
\hline 12 & $\begin{array}{l}\text { Dimova } \\
(2016) 12\end{array}$ & $\begin{array}{l}\text { B-MVQOLI- } \\
15\end{array}$ & 16.44 & $\begin{array}{l}\text { Patients with HD have } \\
\text { low QoL score. }\end{array}$ & NA \\
\hline 13 & $\begin{array}{r}\text { Saputri } \\
\text { (2018)13 }\end{array}$ & $\begin{array}{l}\text { KDQO- } \\
\text { SFTM }\end{array}$ & 55,70 & $\begin{array}{l}\text { Role with the limited physical } \\
\text { suffering of the patient with CKD } \\
\text { disease }\end{array}$ & NA \\
\hline 14 & $\begin{array}{l}\text { Costa } \\
(2016) 38\end{array}$ & $\begin{array}{l}\text { WHOQOL- } \\
\text { BREF }\end{array}$ & 64.96 & $\begin{array}{l}\text { Physical activity affects } \\
\text { QoL of CKD Patients }\end{array}$ & NA \\
\hline 15 & \begin{tabular}{|c|} 
Poppe \\
(2013)15
\end{tabular} & SF-36 & 52.43 & $\begin{array}{llll}\text { CKD } & \text { Influence } & & \text { Qo } \\
\text { patien } & & \text { L } & \\
\text { ts } & & & \end{array}$ & \begin{tabular}{|l|} 
HT,DM, \\
Cardiovaskuler,
\end{tabular} \\
\hline 16 & $\begin{array}{l}\text { Knudsen } \\
\text { (2016)16 }\end{array}$ & KDQOL & 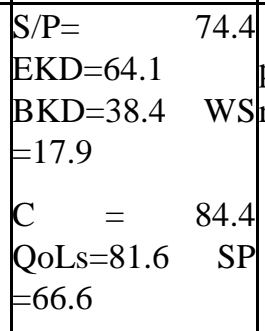 & 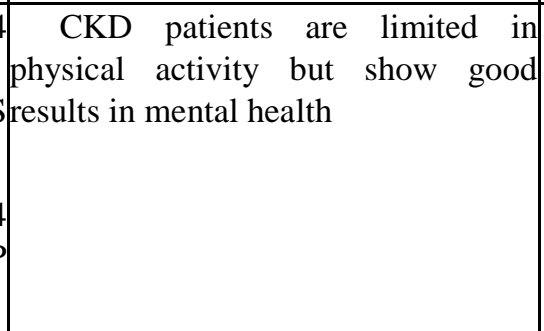 & NA \\
\hline
\end{tabular}




\begin{tabular}{|c|c|c|c|c|c|}
\hline & & & $\mathrm{SS}=74.5$ & & \\
\hline 17 & $\begin{array}{l}\text { Veerappa } \\
\mathrm{n} \\
(2020) 17\end{array}$ & $\begin{array}{l}\text { KDQOL- } \\
\text { SF36 }\end{array}$ & $\mathrm{NA}$ & $\begin{array}{l}\text { Comorbid and age } \\
\text { affect the patient's QoL }\end{array}$ & $\begin{array}{l}\text { Cardiovaskuler. } \\
\text { HT, DM }\end{array}$ \\
\hline 18 & $\begin{array}{l}\text { Naderifar } \\
(2019) 18\end{array}$ & KDQOL-SF & 46.43 & $\begin{array}{l}\text { Compliance with taking } \\
\text { medication and } \\
\text { Age affects QoL }\end{array}$ & NA \\
\hline 19 & $\begin{array}{l}\text { Ossama } \\
(2020) 19\end{array}$ & KDQOL-SF & 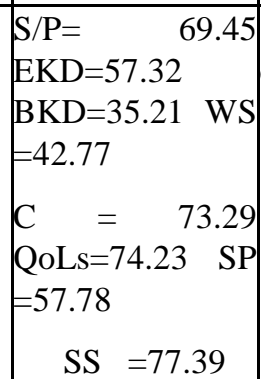 & $\begin{array}{l}\text { HD patients have low QoL, a } \\
\text { comorbid affect QoL }\end{array}$ & $\begin{array}{l}\text { DM, AN } \\
\text { Hepatiti, IH, HT }\end{array}$ \\
\hline 20 & $\begin{array}{r}\text { Rostami } \\
(2013) 20\end{array}$ & KDCF-SF & 51.12 & $\begin{array}{l}\text { Age affects QoL. Physical } \\
\text { limitations are more limited than } \\
\text { health } \\
\text { mentally. }\end{array}$ & NA \\
\hline 21 & $\begin{array}{l}\text { Pan } \\
(2018) 21\end{array}$ & $\begin{array}{l}\text { KDQOL- } \\
\text { SF6D }\end{array}$ & Utility $=0.75$ & $\begin{array}{l}\text { Age and complications } \\
\text { affect QoL }\end{array}$ & Kardiovaskuler \\
\hline 22 & $\begin{array}{l}\text { Nayana } \\
(2016) 22\end{array}$ & KDQOL & $\begin{array}{l}\mathrm{S} / \mathrm{P}=\quad 68.69 \\
\mathrm{EKD}=46.32 \\
\mathrm{BKD}=33.50 \mathrm{C}= \\
61.86 \\
\mathrm{QoLs}=67.56 \quad \mathrm{SP} \\
=65.20 \\
\quad \mathrm{SS}=73.54\end{array}$ & $\begin{array}{l}\text { CKD influences patient's QoL } \\
\text { (age, status, and duration) }\end{array}$ & NA \\
\hline 23 & $\begin{array}{l}\text { Bender } \\
(2018) 23\end{array}$ & $\begin{array}{l}\text { KDQOL- } \\
\text { SF12 }\end{array}$ & NA & HD patients havethe QoL lower & NA \\
\hline 24 & $\begin{array}{l}\text { Sihombin } \\
\text { g }(2017) 24\end{array}$ & KDQOL & 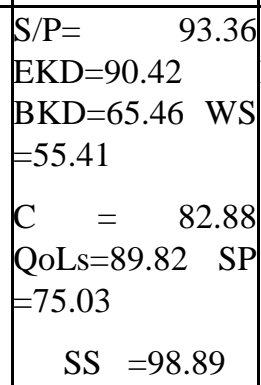 & $\begin{array}{l}\text { CKD affects the physical } \\
\text { limitations of CKD patients. }\end{array}$ & NA \\
\hline 25 & $\begin{array}{l}\text { Morales } \\
\text { (2018) }\end{array}$ & KINDL & 60.39 & $\begin{array}{l}\text { The physical domain is scored } \\
\text { the lowest }\end{array}$ & NA \\
\hline
\end{tabular}




\begin{tabular}{|c|c|c|c|c|c|}
\hline 26 & $\begin{array}{l}\text { Surendra } \\
(2019)\end{array}$ & EQ-5D-3L & Utility $=0.854$ & $\begin{array}{l}\text { HD patients have the QoL } \\
\text { lower }\end{array}$ & NA \\
\hline 27 & $\begin{array}{l}\text { Montilla } \\
\text { (2016) }\end{array}$ & SF-36 & 58.63 & $\begin{array}{c}\text { The quality of life for } \\
\text { hemodialysis patients is moe Low }\end{array}$ & NA \\
\hline 28 & $\begin{array}{l}\text { Tilaki } \\
(2020)\end{array}$ & SF-36 & $\begin{array}{l}44.77 \\
37.1\end{array}$ & $\begin{array}{l}\text { Age affects QoL } \\
\text { CKD patients }\end{array}$ & NA \\
\hline 29 & $\begin{array}{l}\text { Pardede } \\
(2019)\end{array}$ & PedsQL & NA & $\begin{array}{l}\text { CKD affects QoL (duration of } \\
\text { diagnosis, Stage) }\end{array}$ & NA \\
\hline 30 & $\begin{array}{l}\text { Stein } \\
(2012)\end{array}$ & $\begin{array}{l}\text { KDQOL-SF- } \\
36\end{array}$ & $\begin{array}{l}39.14 \\
51.9\end{array}$ & $\begin{array}{l}\text { Patients with Comorbids } \\
\text { have more QoL } \\
\text { low, limited to } \\
\text { physical and mental health }\end{array}$ & $\overline{\mathrm{DM}}$ \\
\hline 31 & $\begin{array}{l}\text { Wang } \\
\text { (2012) }\end{array}$ & $\begin{array}{l}\text { KDQOL-SF- } \\
36\end{array}$ & $\begin{array}{l}\mathrm{PF}=58.8 \\
\mathrm{PRF}=66.8 \\
\mathrm{P} \quad=76.6 \\
\mathrm{GH}=49.4 \\
\mathrm{~V}=58.9 \\
\mathrm{ER}=74.8 \\
\mathrm{MH}=71.7\end{array}$ & $\begin{array}{l}\text { Low CKD patient QoL, } \\
\text { HD duration affects QoL } \\
\text { across ethnicities. }\end{array}$ & NA \\
\hline 32 & $\begin{array}{l}\text { Fructuoso } \\
(2011)\end{array}$ & $\begin{array}{l}\text { KDQOL-SF- } \\
36\end{array}$ & $\begin{array}{l}\mathrm{S} / \mathrm{P}=71.82 \\
\mathrm{EKD}=59.38 \\
\mathrm{BKD}=26.35 \\
\mathrm{WS}=19.85 \\
\mathrm{C}=68.83 \\
\mathrm{QoLs}=80.18 \\
\mathrm{SP}=58.68 \\
\mathrm{SS}=75.05\end{array}$ & $\begin{array}{l}\text { Age, Gender, stage CKD } \\
\text { affect QoL. } \\
\text { Physical health is the } \\
\text { lowest domain and } \\
\text { affect life } \\
\text { patients at CKD stage 1-4 }\end{array}$ & $\mathrm{NA}$ \\
\hline 33 & $\begin{array}{l}\text { Zyoud } \\
(2016)\end{array}$ & EQ-5D-5L & $0.37-59.38$ & $\begin{array}{l}\text { Comorbidity, HD duration } \\
\text { affect QoL }\end{array}$ & $\begin{array}{l}\text { Kardiovaskuler, } \\
\text { DM, HT. }\end{array}$ \\
\hline 34 & $\begin{array}{l}\text { Zimbudzi } \\
\text { (2016) }\end{array}$ & EQ-5D-5L & 0.7 & $\begin{array}{l}\text { CKD patients are limited } \\
\text { self care }\end{array}$ & $\mathrm{NA}$ \\
\hline 35 & $\begin{array}{l}\text { Negi } \\
(2019)\end{array}$ & 36 KDQOL-SF- & 59.28 & $\begin{array}{l}\text { Age, Gender, CKD stage, } \\
\text { presence of comorbidities } \\
\text { affect QoL }\end{array}$ & $\begin{array}{l}\text { Kardiovaskuler, } \\
\text { DM, HT. }\end{array}$ \\
\hline 36 & $\begin{array}{l}\text { Lee } \\
(2016)\end{array}$ & EQ-5D-5L & Utility $=0.87$ & $\begin{array}{l}\text { Stage CKD affects } \\
\text { QoL, the patient feels depressed }\end{array}$ & $\mathrm{NA}$ \\
\hline
\end{tabular}




\begin{tabular}{|c|c|c|c|c|c|}
\hline 37 & $\begin{array}{l}\text { Alqahtani } \\
\text { (2019) }\end{array}$ & KDQOL-SF & $\begin{array}{l}37.4 \\
43.5\end{array}$ & $\begin{array}{l}\text { Increasing age, QoL } \\
\text { Drop }\end{array}$ & DM, HT \\
\hline 38 & $\begin{array}{l}\text { Teles } \\
(2018)\end{array}$ & SF-36 & $\begin{array}{l}\mathrm{PF}=62.6 \\
\mathrm{PRF}=41.6 \\
\mathrm{P} \quad=55 \\
\mathrm{GH}=45.8 \\
\mathrm{SR}=73.4 \\
\mathrm{ER}=59.7 \\
\mathrm{MH}=73.5 \\
\mathrm{~V}=62.4\end{array}$ & $\begin{array}{l}\text { Patients undergoing HD + } \\
\text { comorbidities have a QOL } \\
\text { which is low by prevalence } \\
\text { high depression. }\end{array}$ & $\begin{array}{l}\text { Kardiovaskuler, } \\
\text { DM, HT. }\end{array}$ \\
\hline
\end{tabular}

$\mathrm{NA}=$ Not available; $\mathrm{HT}=$ hipertensi; $\mathrm{DM}=$ Diabetes Melitus; $\mathrm{CG}=\mathrm{Chronic}$ glomerulonephritis; $\mathrm{CP}=\mathrm{Chronic}$ pyelonephritis; $\mathrm{GH}=$ General Health; $\mathrm{PF}=$ Physical functioning; $\mathrm{PRF}=$ Physical role functioning; $\mathrm{P}=\mathrm{Pain}$; V=Vitality; SR= Social role functioning;ER=Emotional role functioning; $\mathrm{MH}=$ Mental Health; $\mathrm{IH}=$ Ischemic Heart; $\mathrm{AN}=\mathrm{Anemia} ; \mathrm{S} / \mathrm{P}=\mathrm{Sym}$ tomps/Problems; $\mathrm{EKD}=$ effect of Kidney Disease; $\mathrm{BKD}=$ Burden of Kidney Disease; WS $=$ Work status; $\mathrm{C}=\operatorname{cognitive}$; QoLs= Quality of social interactio; S=Sleep; SS=Social support. RQ1: Perception of quality of life; RQ2: Satisfaction with health; DF: Physical domain; DP domain Psychological; DRS social relations domain; DMA domain Environment; PU=Peptic ulcer; RT= Rheumatologic; $\mathrm{CPD}=$ Cardiac and Pulmonary Disease;HD= Hemodyalisis; PD; Peritonyal dyalisis;HP=hypoalbuminemia. 
Table 3 shows that in addition to age, the presence of comorbids and disease stage of kidney has an impact on deterioating quality of life. Our review found that $39.4 \%$ of patients with kidney failure were comorbid. Diabetes Mellitus is a type of comorbid that most patients suffer. Teles' research (2018) found 38 scores obtained by kidney failure patients with comorbid Diabetes Mellitus are lower than without Diabetes Mellitus, it is said that low quality of life is followed by high depression due to complications suffered and the large number of drugs that must be consumed which makes financial situation it got worse. Most patient with severe stage CKD feel their quality of life is getting worse and out of control.In this systematic review it was reported that patients consisted of many stages. Fructuso's research (2011) that the QoL score in each domain, both physical health, mental health and social environment, decreased from stage 1 to stage 4 . It can be said that the more severe the stage of the disease, the lower the score. The physical, emotional and social conditions at each stage of the disease are of course different. Patients who were in the final stage had more treatment, more varied amounts of drug consumed, more frequent hemodyalysis duration and longer hospital stay. Osama's study showed that patients with hemodyalysis duration (> 6 years) had lower scores than patients with shorter duration of hemodyalysis. Similar results are shown in Negi (2019) 35's research, the more HD duration is carried out, the worse the QoL value. Patients undergoing hemodyalysis treatment describe a declining quality of life because it is associated with poor financial results due to high funding for one hemodyalysis treatment. Some patients feel dependent on hemodyalysis machines to survive, which makes physical activity decrease over time.This systematic review can provide information for readers and medical personnel in the healt service for more intensive and sustainnable health care towards chronic kidney failure.The results we get can be used as a reference for paramedics in renal failure patient care services to be able to provide more intensive care for patients, to help patients manage comorbidities and complications experienced. It is necessary to train health personnel who are trained and familiar in patient care from the initial stage to patients with the severe late stage. Moral support and family support are important and meaningful for managing mental and emotional management of chronic kidney failure patients.

\section{CONCLUSION}

The result of the systematic reveiw show that the quality of life of the choronic mental failures shows a low value in each domain, especially in the physical and psychological domains. The decrease in quality of life in the physical domain is due to decreased organ fuctions in the patient. Short Form-36 (SF-36) and Kidney Disease Quality of Life (KDQoL) are the most used instrument in measuring the quality of life for chronic kidney failure. Low followed by pateints with comorbid diabetes mellitus. The higher the stage of kidney disease, the worse the pateint's qulity of life wiil be.

\section{REFERENCES}

[1] Cruz MC, Andrade IC, Urrutia IM, Draibe IS, Anto IL. Quality of life in patients with chronic kidney disease. 2011;66(6):991-995. doi:10.1590/S180759322011000600012

[2] Stage E, Disease R, Turlapati S, Nagar N, Pradesh A. EVALUATION OF HEALTH RELATED QUALITY OF LIFE IN HAEMODIALYSIS PATIENTS. 2015;6(11):4733-4742. doi:10.13040/IJPSR.09758232.6(11).4733-42

[3] Voskamp PWM, Diepen M Van, Evans M, et al. The impact of symptoms on health-related quality of life in elderly pre-dialysis patients : effect and importance in the EQUAL study. 2018;(June):1-9. doi:10.1093/ndt/gfy167

[4] Michel RB. Quality of life in hemodialysis patients and the relationship with. 2016:411-420. doi:10.5935/01012800.20160066

[5] Pagels AA, Söderkvist BK, Medin C, Hylander B, Heiwe S. Health-related quality of life in different stages of chronic kidney disease and at initiation of dialysis treatment. 2012:1-11.

[6] Jesus NM, Souza GF De, Mendes-rodrigues C, Pereira O, Neto DA, Cunha CM. Quality of life of individuals with chronic kidney disease on. 2018:364-374. doi:10.1590/2175-8239-JBN-2018-0152

[7] Murali R, Sathyanarayana D, Ma M. ASSESSMENT OF QUALITY OF LIFE IN CHRONIC KIDNEY DISEASE PATIENTS USING THE KIDNEY DISEASE QUALITY OF LIFE-SHORT FORM TM QUESTIONNAIRE IN INDIAN POPULATION : A COMMUNITY BASED STUDY. 2015;8(1):20132016.

[8] Ayanda KA, Abiodun OA, Ajiboye PO. Quality of Life of Chronic Kidney Disease Patients in a Nigerian Teaching Hospital. 2014;4(5).

[9] Tannor EK, Norman BR, Adusei KK, Sarfo FS, Davids MR, Bedu-addo G. Quality of life among patients with moderate to advanced chronic kidney disease in Ghana - a single centre study. 2019:1-10.

[10] Wakeel J Al, Harbi A, Bayoumi M, Al-suwaida K, Ghonaim A. dialysis patients in Saudi Arabia. 2012:570-574. doi:10.5144/0256-4947.2012.570

[11] Abdelghany MA, Elgohary EE, Nienaa YA. Journal of Nephrology \& Therapeutics Assessment of HealthRelated Quality of Life in Patients Receiving Regular Hemodialysis. 2016;6(2):2-5. doi:10.4172/21610959.1000246

[12] Dimova R, Keskinova D, Tzekov V, Ginova-noncheva G. Health-related quality of life in end-stage renal 
disease patients, using the Missoula-Vitas quality of life index : a multicenter study. 2019;92(4):374-381. doi:10.15386/mpr-1320

[13] Saputri VW, Sitorus RJ, Zulkarnain HM. The Quality of Life Chronic Renal Failure ( CRF ) Patients in Hemodialysis Unit at District General Hospital Pringsewu Regency Lampung Province in 2018. 2018;9:1-8.

[14] Morais G, Pinheiro A, Gomes MB, et al. Quality of life of patients with chronic kidneydisease undergoing hemodialysis. :87-99.

[15] Poppe C, Crombez G, Hanoulle I, Vogelaers D, Petrovic M. Improving quality of life in patients with chronic kidney disease : in $\mathrm{fl}$ uence of acceptance and personality. 2013;(July 2012):116-121. doi:10.1093/ndt/gfs151

[16] Knudsen SDP, Eidemak I, Molsted S. Health related quality of life in 2002 and 2015 in patients undergoing hemodialysis : a single center study. 2016;6049. doi:10.1080/0886022X.2016.1207051

[17] Veerappan I, Arvind RM, Ilayabharthi V. Predictors of quality of life of hemodialysis patients in India. 2012;22(1). doi:10.4103/0971-4065.91185

[18] Naderifar M, Tafreshi MZ, Ilkhani M, Akbarizadeh MR. Correlation between quality of life and adherence to treatment in hemodialysis patients. 2019;8(1):22-27. doi:10.15171/jrip.2019.05

[19] Wassef OM, Gendy MF El, Anwar RM El, Taher SM El, Hani BM. Assessment of health - related quality of life of hemodialysis patients in Benha City , Qalyubia Governorate. 2019:1414-1421. doi:10.4103/mmj.mmj

[20] Rostami Z, Einollahi B, Lessan-pezeshki M, et al. Health-Related Quality of

[21] Life in Hemodialysis Patients : An Iranian Multi-Center Study. 2013;5(4):901- 912.

[22] Pan C, Zhou H, Xu B, Wang P. Health-Related Quality of Life and Its Factors of Hemodialysis Patients in. 2018:327-333. doi:10.1159/000485962

[23] Nayana SA, Balasubramanian T, Nathaliya PM, et al. ScienceDirect A cross sectional study on assessment of health related quality of life among end stage renal disease patients undergoing hemodialysis. Clin Epidemiol Glob Heal. 2017;5(3):148-153. doi:10.1016/j.cegh.2016.08.005

[24] Aguiar R, Pei M, Rashid A, Bengt Q. Health - related quality of life in peritoneal dialysis patients : A narrative review. 2018:1-11. doi:10.1111/sdi.12770

[25] Sihombing J, Hakim L, Andayani TM, Irijanto F. QUALITY OF LIFE OF CHRONIC KIDNEY DISEASE PATIENTS WITH ROUTINE
HEMODIALYSIS IN GENERAL HOSPITALS IN SLEMAN YOGYAKARTA. 2017;9(2).

[26] Morales P, Loza R, Vasquez J, Baique P, Reyes M. Quality of Life of Children with Chronic Kidney Disease Undergoing Renal Replacement Therapy. 2018;4(4). doi:10.4172/2472-1220.1000173

[27] Surendra NK, Rizal M, Manaf A, et al. Health related quality of life of dialysis patients in Malaysia : Haemodialysis versus continuous ambulatory peritoneal dialysis. 2019:1-10.

[28] Perales CM, Duschek S, Reyes GA. Original article Health-related quality of life in chronic kidney desease : Predictive relevance of mood and somatic symptoms ฉ. 2016;6(3):275-282.

[29] Heidari B. of Kidney Diseases and Transplantation Renal Data from Asia -

[30] Africa A Comparison of Health-related Quality of Life in Patients with Renal Failure under Hemodialysis and Healthy Participants. 2017;28(1):133-140.

[31] Pardede SO, Rafli A, Gunardi H. of Kidney Diseases and Transplantation Original Article Quality of Life in Chronic Kidney Disease Children Using Assessment Pediatric Quality of Life Inventory TM. 2019;30(4):812-818.

[32] Stein DM, Welch JL, Kraus MA, Slaven JE. HealthRelated Quality of Life Among Patients Receiving Home Dialysis Therapies. 2012:10-18.

[33] Wang V, Seow Y, Chow WL. Influence of ethnicity on health-related quality of life of hemodialysis patients in Singapore. 2012;35:217-225. doi:10.5301/ijao.5000014

[34] Fructuoso MR, Castro R, Prata C, Morgado T. Quality of life in chronic kidney disease. 2011:91-96. doi:10.3265/Nefrologia.pre2010.Jul.10483

[35] Zyoud SH, Daraghmeh DN, Mezyed DO, et al. Factors affecting quality of life in patients on haemodialysis : a cross-sectional study from Palestine. BMC Nephrol. 2016:1-12. doi:10.1186/s12882-016-0257-z

[36] Zimbudzi E, Samlero R, Kerr PG, Zoungas S. How much is enough? An investigation of the relationship between haemodialysis adequacy and quality of life of elderly patients. 2016;21:314-320. doi:10.1111/nep.12594 Res IJA. QUALITY OF LIFE OF CHRONIC KIDNEY DISEASE PATIENTS UNDERGOING MAINTENANCE Manuscript Info Abstract ISSN : 2320-5407 Introduction : 2018;7(9):1131-1135. doi:10.21474/IJAR01/9764

[37] Lee SJ, Son H. Comparison of health-related quality of life between patients with stage 3 and 4 chronic kidney disease and patients undergoing continuous ambulatory 
peritoneal dialysis. 2016;5:166-173. doi:10.1111/jjns.12101

[38] Alqahtani NA, Al- MA, Al-alsheikh K, Helmy W. Quality of life among patients with chronic renal failure on hemodialysis at the military hospital in southern region of Saudi Arabia. 2019;6(5):6-9. doi:10.15406/mojap.2019.06.00265

[39] Teles F, Letícia A, Albuquerque A De, et al. Quality of life and depression in haemodialysis patients. Psychol Health Med. 2018;8506:1-10. doi:10.1080/13548506.2018.1469779

[40] World Health Organization. 2016. Management of substance abuse: WHO Quality of Life-BREF (WHOQOL-BREF). http://www/who.int (Diakses 23 Juni 2020).

[41] Wyld MLR, Morton RL, Clayton P, et al. The impact of progressive chronic kidney disease on health-related quality-of-life : a 12-year community cohort study. Qual Life Res. 2019;(0123456789). doi:10.1007/s11136-019-02173-1

[42] 41 Shan S, Saleem C, Mawed A, Unruh M. HealthRelated Quality of Life in End- Stage Renal Disease Patients : How Often Should We Ask and What Do We Do with the Answer ? 2016;87106:218-224. doi:10.1159/000441462 